\title{
Channel Parameters Identification Based on IMM Algorithm for Variant Correlation Channel
}

\author{
Zhuo Yang, ${ }^{1}$ Yiru Inoue, ${ }^{2}$ Jian Wan, ${ }^{3}$ and Lei Chen ${ }^{1}$ \\ ${ }^{1}$ National Police University of China, Shenyang 110854, China \\ ${ }^{2}$ Envisage Information Systems, Ithaca, NY 14850, USA \\ ${ }^{3}$ College of Information and Communication, Harbin Engineering University, Harbin 150001, China \\ Correspondence should be addressed to Zhuo Yang; yangzhuo666@gmail.com
}

Received 29 January 2015; Accepted 13 March 2015

Academic Editor: Francesco Tornabene

Copyright (C) 2015 Zhuo Yang et al. This is an open access article distributed under the Creative Commons Attribution License, which permits unrestricted use, distribution, and reproduction in any medium, provided the original work is properly cited.

In wireless communication systems, correct knowledge of the correlation of a fading channel is essential for channel estimation. Both the reliability of the estimated channel impulse response (CIR) and the adjustment of an adaptive communication system need the accurate correlation information, which is difficult to identify especially when changing. By modeling the fading channel as a hybrid dynamic system, a channel estimation algorithm based on Interacting Multiple Model (IMM) is presented with the consideration of time-variant channel correlation. Applying the IMM algorithm, the proposed channel estimator can identify the channel correlation. With the accurate information of channel correlation, the proposed algorithm is capable of performing accurate estimation on the fading wireless channel with time-variant or time-invariant correlation. Our simulations demonstrate that the IMM based channel estimation algorithm has good performance in estimating CIR as well as in identifying the channel correlation.

\section{Introduction}

Information transmission with high data rates and reliable performance is required in wireless communication systems. However, the performance of the communication systems suffers from the signal distortion caused by wireless channel. As a fundamental technology to ensure communication performance, a channel estimation algorithm is required to measure the channel parameters and reduce the influence on the communication system.

In the application of wireless control and wireless sensor systems, high performance communication between maneuvering objects is required. Obviously, the impulse response of the channel is time-variant because of the Doppler effect. Furthermore, the channel correlation which governs the way that the channel varies is also time-variant because of the frequent change in the speed of the moving object. This scenario can be treated as a channel that varies in different modes. In this case, the performance of channel estimation is affected not only by the variance of channel impulse response but also by the changing of channel modes.
In the adaptive communication systems, system parameters, signal modes, and transmission modes can be adjusted according to the channel quality. Therefore, the statistical information of the channel, such as channel correlation, reflecting how the channel changes, is required at each moment to adjust communication parameters. Without the channel statistical information directly provided by the channel estimator, the system parameters can only be adjusted according to the channel information calculated indirectly, for example, bit error rate (BER). Therefore, tracking the channel correlation is important for the channel estimation algorithm to achieve good performance. Channel estimation algorithms that provide CIR as well as the statistical information should be developed. This has not been systematically studied.

Among channel estimation algorithms, Linear Minimum Mean Square error (LMMSE) is widely used, since it is optimum in minimizing the Mean Square Error (MSE) of the estimated channel parameters in the presence of Additive White Gaussian Noise (AWGN). It is shown that LMMSE is very attractive for the channel estimation in [1-3]. However, 
the computational complexity of LMMSE is very high due to the large amount of information operated comprehensively in the estimation algorithm.

The Kalman filter (KF), as an approximation of the Optimal Bayesian filter, is used in a wide range of engineering and econometric applications because of its high accuracy and efficiency on parameter estimating in dynamic systems [4]. In wireless channel estimation, the Kalman filter has been applied with the wireless channel described by autoregressive (AR) model [5-7]. These methods are functional with low computational complexity and the ability of fast tracking the varying channel in the situation that the speed of the channel fading is constant.

In the case of a maneuvering receiver, channel correlation varies into different modes because of the changing Doppler shift corresponding to the relative speed between the transmitter and the receiver. Without information on channel correlation, traditional channel estimation algorithms are limited in performance in such situations, because they cannot effectively respond to the changes in channel mode $[8,9]$.

The Multiple Model (MM) filter was developed to solve the problem of system mode changes [4]. The Interacting Multiple Model (IMM) algorithm is one of the most efficient approaches among MM algorithms and is widely used in object tracking [10] and other hybrid dynamic systems [11, 12]. In communication systems the IMM algorithm can provide accurate channel parameters by applying two Kalman filters to the static and moving states of the receiver [13].

The contribution of this paper is to propose a new channel estimation algorithm based on the IMM algorithm. This algorithm characterizes the fading channel using state-space models and describes the dynamic channel correlation with multiple models. The proposed method is capable of tracking the CIR and identifying the channel correlation which changes according to the maneuverability of the receiver.

This paper is organized as follows. The signal transmission of a communication system as well as the Rayleigh fading multipath wireless channel is modeled mathematically in Section 2. In Section 3, the processes of channel correlation identification and channel estimation based on IMM theory are presented with theoretical proof after the introduction of the KF. The importance of channel correlation identification and the applicability of the proposed algorithm are also discussed in Section 3. After presenting and analyzing a number of simulation results in Section 4, the conclusions are summarized in Section 5.

\section{Problem Formulation}

2.1. Transmission System Model. The transform and transmission of data symbols are illustrated in Figure 1. In the transmitter, a sequence composed of binary data $b[j]$ is modulated in baseband and transforms into a sequence of complex signals $d[k]$. After being modulated by carrier with frequency $f_{c}, d[k]$ transforms into waveform $s(t)$ and transmitted over a Rayleigh fading channel $h(t, \tau)$, where $\tau$ is the delay and $t$ denotes current time. In the receiver, after carrier demodulation and sampling, $y[k]$ is obtained from the receiving signal and can be expressed as follows:

$$
y[k]=\left.d[l] * h[k, l]\right|_{l=k}+n[k],
$$

where $h[k, l]$ is the discrete time CIR with $k$ and $l$ denoting current time and the delay tab; $s[l]$ is the discrete time receiving signal with $s(t)$ being sampled; $n[k]$ is the sampled noise signal. With the help of channel estimation algorithms, the CIR can be estimated, and the output of the channel estimation algorithm is denoted by $\widehat{h}[k, l]$. Using the information of $\widehat{h}[k, l]$, received signal can be recovered from $y[k]$, and the recovered signal is denoted as $\widehat{d}[i]$. After baseband demodulation, the received data $\widehat{b}[j]$ is obtained from $\widehat{d}[i]$.

2.2. Fading Channel. In wireless channels, with the effect of atmospheric reflection, refraction, and reflections from object such as buildings, the signal may travel in more than one path from the transmitter to the receiver. In mobile communication systems, the attenuation and the delay of the CIR are time-variant. After being sampled with the symbol period $T_{s}$, the CIR of the multipath time-variant channel at time $k$ can be written in discrete time victor form:

$$
\mathbf{h}[k]=\{h[k, 1], \ldots, h[k, l], \ldots, h[k, L]\}^{\mathrm{T}},
$$

where $l$ is the number of the paths and $L$ channel paths have the significant multipath energy. The time delay of each path is $l T_{s} . h[k, l]$ is the complex gain of the $l$ th path in time $k T_{s}$. And the superscript "T" in (2) denotes transpose.

In mobile communication systems, the time-variance of the CIR taps is caused by the motion of the transmitter and/or receiver and is quantified by the maximum Doppler shift. When the number of multiple reflective paths is large and there is no line-of-sight signal component, the envelop of every tap of CIR can be statistically described by a Rayleigh probability density function, and the phases of each tap are uniformly distributed in $[0,2 \pi]$. According to the property of the Rayleigh distribution, the real and imaginary components of each tap of CIR are independently and identically distributed (i.i.d.) zero mean Gaussian. As a result, the CIR at time $k-1$ and time $k$ can be represented as a first-order AR model [5] in the following form:

$$
\mathbf{h}[k]=a \mathbf{h}[k-1]+\mathbf{v}[k],
$$

where $\mathbf{v}[k]$ is the complex noise vector. Making $a=1$, (3) can be written as

$$
\mathbf{h}[k]=\mathbf{h}[k-1]+\mathbf{v}[k] .
$$

The real and imaginary components of each element of $\mathbf{v}[k]$ are zero mean i.i.d. Gaussian. The covariance matrix of $\mathbf{v}[k]$ is determined by the autocorrelation of $\mathbf{h}[k]$. For the wide sense stationary uncorrelated scattering model $[14,15]$, the autocorrelation function of the multipath Rayleigh fading channel is [15]

$$
\begin{aligned}
\mathrm{E}\left\{h\left[k_{1}, l_{1}\right] h^{*}\left[k_{2}, l_{2}\right]\right\}= & c \cdot J_{0}\left(2 \pi f_{\mathrm{d}}\left(k_{1}-k_{2}\right) T_{c}\right) \\
& \cdot e^{-l_{1} / L} \delta\left(l_{1}-l_{2}\right),
\end{aligned}
$$




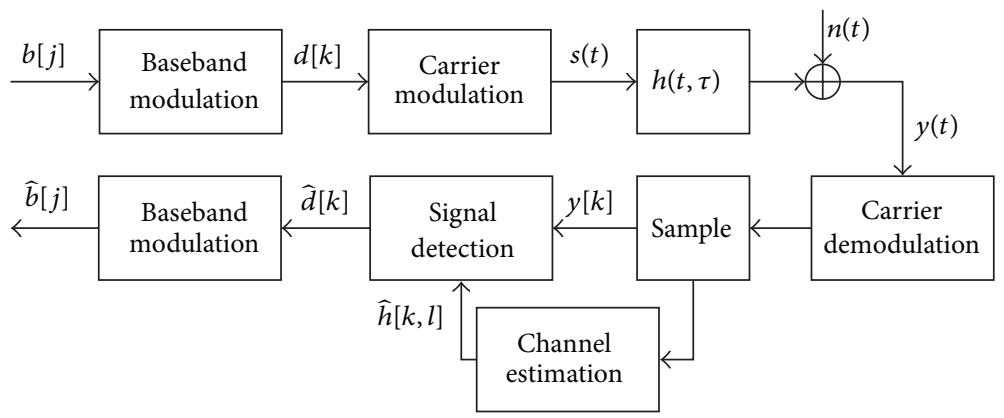

FiguRE 1: Diagram of a communication system.

where the superscript "** denotes the conjugate transposition operation; $c=1 /\left(\sum_{l} e^{-l / L}\right)$ is a normalization constant. $J_{0}(x)$ is the zeroth-order Bessel function of the first kind. The maximum Doppler frequency shift in hertz $f_{\mathrm{d}}$ is shown as follows:

$$
f_{\mathrm{d}}=\frac{v \cdot f_{0}}{C}
$$

where $v$ is the relative speed between the transmitter and the receiver, $f_{0}$ is the emitted frequency, and $C$ denotes the speed of wave.

\section{Interacting Multiple Model (IMM) Based Channel Estimation}

Assuming that the coherence time of the channel is $T_{\text {coh }}$, to estimate the CIR, the same training sequences with the length of $N_{t}$ are sent every $T_{t}$, where $T_{t} \leq T_{\text {coh }}$. The received signal of the $k$ th training sequence can be written in the form of matrix as follows:

$$
\mathbf{y}[k]=\mathbf{D} \cdot \mathbf{h}[k]+\mathbf{w}[k],
$$

where $N_{t} \times 1$ vector, $\mathbf{y}[k]$ denotes the $k$ th received training signal after being sampled and can be defined as

$$
\begin{array}{r}
\mathbf{y}[k]=\left\{y\left[k\left(\frac{T_{t}}{T_{s}}\right)\right], \ldots, y\left[k\left(\frac{T_{t}}{T_{s}}\right)+m\right], \ldots,\right. \\
\left.y\left[k\left(\frac{T_{t}}{T_{s}}\right)+N_{t}-1\right]\right\}^{\mathrm{T}}, \quad 1 \leq m \leq N_{t} .
\end{array}
$$

$\mathbf{w}[k]$ in (7) is the complex noise which distributes independently with zero means in both real and imaginary parts and covariance matrix $\mathbf{R}[k]$. $\mathbf{D}$ in (7) is the transmitted training signal matrix. Assuming that the transmitter sends the same training sequence every time, $\mathbf{D}$ is a constant matrix which can be written as

$$
\mathbf{D}=\left[\begin{array}{cccc}
d[0] & 0 & \cdots & 0 \\
d[1] & d[0] & \cdots & 0 \\
\vdots & \vdots & \ddots & \vdots \\
d[L-1] & d[L-2] & \cdots & d[0] \\
\vdots & \vdots & \ddots & \vdots \\
d\left[N_{t}-1\right] & d\left[N_{t}-2\right] & \cdots & d\left[N_{t}-L\right]
\end{array}\right],
$$

where the element $d[m]$ denotes the training sequence.

Observing (4) and (7), it can be found that they are in the typical form of state-space model of a dynamic system. Equation (4) is the stochastic difference equation. $\mathbf{h}[k]$ is the vector to be estimated of the dynamic system. Vector $\mathbf{v}[k]$ is the process noise with zero mean and covariance $\mathbf{Q}[k]$ which is denoted as

$$
\mathbf{Q}[k]=\mathrm{E}\left[\mathbf{v}[k] \cdot \mathbf{v}^{*}[k]\right] .
$$

Equation (7) is the measurement equation, where $y[k]$ is the measurement of $\mathbf{h}[k]$, measured by matrix $\mathbf{D}$ and polluted by the measurement noise vector $\mathbf{w}[k]$ with covariance $\mathbf{R}[k]$.

3.1. Kalman Filter $(K F)$ Theory. Separating the real and image parts $\mathbf{h}[k]$ and $\mathbf{y}[k]$, they can be written as

$$
\begin{aligned}
& \mathbf{h}_{\mathrm{ex}}[k]=\{\operatorname{Re}\{\mathbf{h}[k]\}, \operatorname{Im}\{\mathbf{h}[k]\}\}^{\mathrm{T}}, \\
& \mathbf{y}_{\mathrm{ex}}[k]=\{\operatorname{Re}\{\mathbf{y}[k]\}, \operatorname{Im}\{\mathbf{y}[k]\}\}^{\mathrm{T}},
\end{aligned}
$$

where $\operatorname{Re}\{\cdot\}$ and $\operatorname{Im}\{\cdot\}$ are the real part and the image part of the complex value. Then (4) and (7) can be written as

$$
\begin{aligned}
& \mathbf{h}_{\mathrm{ex}}[k]=\mathbf{h}_{\mathrm{ex}}[k-1]+\mathbf{v}_{\mathrm{ex}}[k], \\
& \mathbf{y}_{\mathrm{ex}}[k]=\mathbf{D}_{\mathrm{ex}} \cdot \mathbf{h}_{\mathrm{ex}}[k]+\mathbf{w}_{\mathrm{ex}}[k],
\end{aligned}
$$

where $\mathbf{D}_{\mathrm{ex}}$ is denoted as

$$
\mathbf{D}_{\text {ex }}=\left[\begin{array}{cc}
\operatorname{Re}\{\mathbf{D}\} & -\operatorname{Im}\{\mathbf{D}\} \\
\operatorname{Im}\{\mathbf{D}\} & \operatorname{Re}\{\mathbf{D}\}
\end{array}\right] .
$$


The recursion of KF is given by the following equations.

(1) The predicted mean and covariance matrix:

$$
\begin{aligned}
& \widehat{\mathbf{h}}_{\mathrm{ex}}\left[k^{-}\right]=\widehat{\mathbf{h}}_{\mathrm{ex}}[k-1], \\
& \mathbf{P}\left[k^{-}\right]=\mathbf{P}[k-1]+\mathbf{Q}[k] .
\end{aligned}
$$

(2) The predicted measurement, innovation covariance matrix, and Kalman gain:

$$
\begin{aligned}
& \widehat{\mathbf{y}}_{\mathrm{ex}}\left[k^{-}\right]=\mathbf{D}_{\mathrm{ex}} \widehat{\mathbf{h}}_{\mathrm{ex}}\left[k^{-}\right], \\
& \mathbf{D}_{K}[k]=\mathbf{D}_{\mathrm{ex}} \mathbf{P}\left[k^{-}\right] \mathbf{D}_{\mathrm{ex}}^{\mathrm{T}}+\mathbf{R}[k], \\
& \mathbf{K}[k]=\mathbf{P}\left[k^{-}\right] \mathbf{D}_{\mathrm{ex}}^{\mathrm{T}} \mathbf{D}_{K}^{-1}[k] .
\end{aligned}
$$

(3) The posterior mean (estimated value) and covariance matrix:

$$
\begin{aligned}
& \mathbf{u}[k] \triangleq \mathbf{y}_{\mathrm{ex}}[k]-\widehat{\mathbf{y}}_{\mathrm{ex}}\left[k^{-}\right], \\
& \widehat{\mathbf{h}}_{\mathrm{ex}}[k]=\widehat{\mathbf{h}}_{\mathrm{ex}}\left[k^{-}\right]+\mathbf{K}[k] \mathbf{u}[k], \\
& \mathbf{P}[k]=\mathbf{P}\left[k^{-}\right]-\mathbf{K}[k] \mathbf{D}_{\mathrm{ex}} \mathbf{P}\left[k^{-}\right],
\end{aligned}
$$

where “へ” denotes the estimated value; the superscript “-” of the time $k$ denotes the predicted value obtained from the estimated value in time $k-1 ; \mathbf{P}[k]$ is the covariance matrix of $\mathbf{h}_{\mathrm{ex}}[k+1]$ assuming that the mean of $\mathbf{h}_{\mathrm{ex}}[k+1]$ is $\widehat{\mathbf{h}}_{\mathrm{ex}}[k]$.

From the equations of $\mathrm{KF}$, it can be noticed that, to ensure the usability of KF, the covariance matrixes of process noise and measurement noise should be known. However, in real cases, they can only be calculated statistically rather than measured. In that case, noise covariance, say process noise, can be denoted as

$$
\widehat{\mathbf{Q}}[k]=\frac{(k-1) \widehat{\mathbf{Q}}[k-1]+\mathbf{v}[k] \mathbf{v}^{*}[k]}{k} .
$$

With (17), KF can provide an accurate noise covariance when the noise covariance is constant.

3.2. IMM Based Channel Estimation. In hybrid systems, the dynamic parameter to be estimated varies in different modes. In this case, the IMM estimator is one of the best compromises available between complexity and performance, because of its low computational requirements and the accuracy which is almost the same as that of many other algorithms with much higher complexity [10]. As a powerful approach to adaptive estimation in dynamic hybrid systems, IMM estimator is functional of solving problems of structural and parametric changes $[4,11,16]$.

In wireless communications, CIR varies randomly. In addition, the channel features, such as channel correlation, change from time to time. As a result, channel estimation can be achieved by applying IMM estimation method.

(1) Channel Correlation Identification. In mobile communications, the CIR varies with the speed reflected by the channel correlation, which is described in (5). On the one hand, the information of channel correlation is needed by the channel estimator to estimate the CIR accurately. On the other hand, channel correlation is important information for the upper layer of the adaptive communication networks to adapt the signal transmission scheme according to the channel feature. From the channel stochastic difference equation denoted by (4), the process noise vector $\mathbf{v}[k]$ reflects the change of the CIR. And $\mathbf{Q}[k]$, the covariance matrix of $\mathbf{v}[k]$, reflects the channel correlation, which can be derived as

$$
\mathbf{Q}[k]=\left(\mathbf{E}_{h}[k]+\mathbf{E}_{h}^{*}[k]\right)-\left(\mathbf{R}_{h}\left[k, T_{t}\right]+\mathbf{R}_{h}^{*}\left[k, T_{t}\right]\right),
$$

where $\mathbf{R}_{h}\left[k, T_{t}\right]$ and $\mathbf{E}_{h}[k]$ are the correlation matrix and the covariance matrix of CIR and are defined as follows:

$$
\begin{aligned}
\mathbf{R}_{h}\left[k, T_{t}\right] & =\mathrm{E}\left[\mathbf{h}[k] \cdot \mathbf{h}^{*}[k-1]\right], \\
\mathbf{E}_{h}[k] & =\mathrm{E}\left[\mathbf{h}[k] \cdot \mathbf{h}^{*}[k]\right] .
\end{aligned}
$$

The derivation of (18) is shown in Appendix A. Seen from (5), the gains of each tap are independent. In (18), $\mathbf{E}_{h}[k]$ is in the following form:

$$
\mathbf{E}_{h}[k]=\operatorname{diag}\left\{c \cdot e^{0 / L}, \ldots, c \cdot e^{-l / L}, \ldots, c \cdot e^{-(L-1) / L}\right\} .
$$

In practice, $\mathbf{E}_{h}[k]$ can be obtained by using the estimated CIR. The elements of $\mathbf{R}_{h}\left[k, T_{t}\right]$ can be calculated by (5) and be written as

$$
\begin{array}{r}
\mathbf{R}_{h}\left[k, T_{t}\right]=\operatorname{diag}\left\{c \cdot J_{0}\left(2 \pi f_{\mathrm{d}}[k] T_{t}\right) \cdot e^{0 / L}, \ldots,\right. \\
c \cdot J_{0}\left(2 \pi f_{\mathrm{d}}[k] T_{t}\right) \cdot e^{-l / L}, \ldots, \\
\left.c \cdot J_{0}\left(2 \pi f_{\mathrm{d}}[k] T_{t}\right) \cdot e^{-(L-1) / L}\right\},
\end{array}
$$

where $f_{\mathrm{d}}[k]$ is the maximum Doppler frequency shift at time $k$. As $\mathbf{E}_{h}[k]$ and $\mathbf{R}_{h}\left[k, T_{t}\right]$ are both diagonal matrixes, $\mathbf{Q}[k]$ is also diagonal and can be denoted as

$$
\mathbf{Q}[k]=2 \mathbf{E}_{h}[k]-2 \mathbf{R}_{h}\left[k, T_{t}\right] .
$$

Seen from (23), (22), and (6), $f_{\mathrm{d}}$ is caused by the relative speed between the transmitter and the receiver and governs the channel correlation, which reflects the varying speed of the CIR.

As a result, both $\mathbf{Q}[k]$ and $\mathbf{R}_{h}\left[k, T_{t}\right]$ can be identified by establishing multiple models on Doppler shift $f_{\mathrm{d}}$ and conducting IMM estimation based on these models. Models are established by dividing $f_{\mathrm{d}}$ into a series of values which are indexed by $f_{\mathrm{d}}^{(i)}$. When mode $f_{\mathrm{d}}^{(i)}$ is in effect at time $k$, the system mode is denoted as

$$
\begin{gathered}
f_{\mathrm{d}}^{(i)}[k] \triangleq\left\{f_{\mathrm{d}}[k]=f_{\mathrm{d}}^{(i)}\right\}, \\
i \in\left\{1,2, \ldots, N_{f}\right\}, \quad \forall f_{\mathrm{d}}^{(i)} \in \mathbf{M},
\end{gathered}
$$

where $\mathbf{M}$ is the set of all modal states. $N_{f}$ is the amount of models in $\mathbf{M}$. Making $\mathbf{Y}^{k}$ the set of all the measurements $\mathbf{y}[k]$ in (7) from the initial time to time $k, \mathbf{Y}^{k}$ is denoted as

$$
\mathbf{Y}^{k}=\{\mathbf{y}[0], \mathbf{y}[1], \ldots, \mathbf{y}[k]\} .
$$


The optimal estimation of $\mathbf{R}_{h}\left[k, T_{t}\right]$ in the MMSE sense is the expectation based on the posterior distribution. The estimated value of $\mathbf{R}_{h}\left[k, T_{t}\right]$ is given by

$$
\begin{aligned}
\widehat{\mathbf{R}}_{h}\left[k, T_{t}\right] & \triangleq \mathrm{E}\left[\mathbf{R}_{h}\left[k, T_{t}\right] \mid \mathbf{Y}^{k}\right] \\
& =\sum_{i} \mathrm{E}\left[\mathbf{R}_{h}\left[k, T_{t}\right] \mid \mathbf{Y}^{k}, f_{\mathrm{d}}^{(i)}[k]\right] \cdot \operatorname{Pr}\left\{f_{\mathrm{d}}^{(i)}[k] \mid \mathbf{Y}^{k}\right\} \\
& =\sum_{i} \widehat{\mathbf{R}}_{h}^{(i)}\left[k, T_{t}\right] \cdot \mu^{(i)}[k],
\end{aligned}
$$

where $\widehat{\mathbf{R}}_{h}^{(i)}\left[k, T_{t}\right]$ is the estimated value of $\mathbf{R}_{h}\left[k, T_{t}\right]$ based on model $i$ and is defined as

$$
\widehat{\mathbf{R}}_{h}^{(i)}\left[k, T_{t}\right] \triangleq \mathrm{E}\left[\mathbf{R}_{h}\left[k, T_{t}\right] \mid \mathbf{Y}^{k}, f_{\mathrm{d}}^{(i)}[k]\right] ;
$$

$\mu^{(i)}[k]$ is the posterior probability that model $i$ is affected at time $k$ and is given by

$$
\mu^{(i)}[k] \triangleq \operatorname{Pr}\left\{f_{\mathrm{d}}^{(i)}[k] \mid \mathbf{Y}^{k}\right\} .
$$

In (26), $\widehat{\mathbf{R}}_{h}^{(i)}\left[k, T_{t}\right]$ can be calculated by (22) using the value of $f_{\mathrm{d}}$ in each model. Consequently, the problem of $\mathbf{R}_{h}\left[k, T_{t}\right]$ identification is reduced to the problem of calculating $\mu^{(i)}[k]$, which is solved in the following sections.

(2) Channel Estimation. Seen from the discussion above, multiple models are established on Doppler shift $f_{\mathrm{d}}$. The mode changes are governed by a Markov chain with the transition probability matrix $\Gamma$, whose element $\Gamma^{(j i)}$ is denoted as

$$
\Gamma^{(j i)}=\operatorname{Pr}\left(f_{\mathrm{d}}^{(i)}[k] \mid f_{\mathrm{d}}^{(j)}[k-1]\right) .
$$

Seen from (29), $\Gamma^{(j i)}$ is the transition probability from mode $j$ in time $k-1$ to mode $i$ in time $k$ and can be obtained statistically. The optimal estimation of CIR can be denoted as

$$
\begin{aligned}
\widehat{\mathbf{h}}[k] & \triangleq \mathrm{E}\left[\mathbf{h}[k] \mid \mathbf{Y}^{k}\right] \\
& =\sum_{i} \mathrm{E}\left[\mathbf{h}[k] \mid \mathbf{Y}^{k}, f_{\mathrm{d}}^{(i)}[k]\right] \cdot \operatorname{Pr}\left\{f_{\mathrm{d}}^{(i)}[k] \mid \mathbf{Y}^{k}\right\} \\
& =\sum_{i} \widehat{\mathbf{h}}^{(i)}[k] \cdot \mu^{(i)}[k],
\end{aligned}
$$

where $\widehat{\mathbf{h}}^{(i)}[k]$ is the estimated value of $\mathbf{h}[k]$ based on model $i$ and is defined as

$$
\widehat{\mathbf{h}}^{(i)}[k] \triangleq \mathrm{E}\left[\mathbf{h}[k] \mid \mathbf{Y}^{k}, f_{\mathrm{d}}^{(i)}[k]\right]
$$

$\mathbf{Y}^{k}$ and $\mu^{(i)}[k]$ are defined by (25) and (28). In optimal Bayes' theorem,

$$
\begin{aligned}
\mu^{(i)}[k] & =\operatorname{Pr}\left\{f_{\mathrm{d}}^{(i)}[k] \mid \mathbf{Y}^{k}\right\} \\
& =\operatorname{Pr}\left\{f_{\mathrm{d}}^{(i)}[k] \mid \mathbf{y}[k], \mathbf{Y}^{k-1}\right\} \\
& =\frac{\operatorname{Pr}\left\{\mathbf{y}[k] \mid f_{\mathrm{d}}^{(i)}[k], \mathbf{Y}^{k-1}\right\} \cdot \mu^{(i)}\left[k^{-}\right]}{\operatorname{Pr}\left\{\mathbf{y}[k] \mid \mathbf{Y}^{k-1}\right\}},
\end{aligned}
$$

where $\operatorname{Pr}\left\{\mathbf{y}[k] \quad \mid f_{\mathrm{d}}^{(i)}[k], \mathbf{Y}^{k-1}\right\}$ is the likelihood function, $\operatorname{Pr}\left\{\mathbf{y}[k] \mid \mathbf{Y}^{k-1}\right\}$ is the normalization factor, and $\mu^{(i)}\left[k^{-}\right] \triangleq$ $\operatorname{Pr}\left\{f_{\mathrm{d}}^{(i)}[k] \mid \mathbf{Y}^{k-1}\right\}$ is the prediction model probability, which can be obtained by

$$
\mu^{(i)}\left[k^{-}\right]=\sum_{j} \Gamma^{(j i)} \cdot \mu^{(j)}[k-1] .
$$

As a result, $\mu^{(i)}[k]$ can be described in the following form:

$$
\mu^{(i)}[k]=\frac{\operatorname{Pr}\left\{\mathbf{y}[k] \mid f_{\mathrm{d}}^{(i)}[k], \mathbf{Y}^{k-1}\right\} \cdot \sum_{j} \Gamma^{(j i)} \cdot \mu^{(j)}[k-1]}{\operatorname{Pr}\left\{\mathbf{y}[k] \mid \mathbf{Y}^{k-1}\right\}} .
$$

Seen from (30) and (34), in optimal Bayes filters, every time CIR is estimated, the history of measurements $\mathbf{Y}^{k}$ is needed. The longer time that the estimator works for, the larger the amount of calculation is required. In view of this, by suboptimally approximating Bayes filter, fixed memory algorithms have been proposed [4, 17]. The generalized pseudo-Bayesian filter of order $r(\mathrm{GPB} r)$ approaches the optimal algorithm taking a memory history of $r-1$ steps into account. Hence, in channel estimation $N_{m}^{r}$ filters are needed in each time $k$. IMM estimator performs as well as GPB2, with the lower computational requirements than GPB1. Thus the IMM algorithm has enjoyed remarkable success as one of the most cost-effective schemes for the estimation in hybrid systems.

The IMM channel estimator is recursive. $N_{f}$ Kalman filters, corresponding to each mode, run in parallel in each cycle, which consist of four steps: interacting/mixing, filtering, and combination. The structure of IMM channel estimator is also shown in Figure 2, where $\widehat{\mathbf{h}}^{(i)}[k], \mathbf{P}^{(i)}[k]$, and $\mathbf{R}_{h}^{(i)}\left[k, T_{t}\right]$ are the estimated CIR by KF $i$ at time $k$, its covariance, and its correlation; $\widehat{\mathbf{h}}^{(0 i)}[k]$ and $\mathbf{P}^{(0 i)}[k]$ are the mixed condition for KF $i$ at time $k$ and its covariance; $\widehat{\mathbf{h}}[k]$, $\mathbf{P}[k]$, and $\mathbf{R}_{h}\left[k, T_{t}\right]$ are the combined estimated CIR at time $k$, its covariance, and its correlation; $\mu^{(i)}\left[k^{-}\right]$and $\mu^{(i)}[k]$ are the predicted and posterior probabilities for $\mathrm{KF} i ; \lambda^{(i)}[k]$ is the likelihood function of KF $i$. In each cycle, the estimated CIR and model probability $\mu^{(i)}[k]$ are firstly interacted with each other and mixed for each mode under the assumption that this mode is in effect at the current time. Therefore the initial condition for each filter based on certain model is obtained. Secondly, the interacted/mixed initial conditions are sent to their particular model-based filters. In channel estimation, KFs based on different models are applied in the filtering step. The estimated CIRs and the updated model probabilities based on each model are obtained. After the filtering step, the outputs of each filter are combined as the final result at the current time. Seen from the upper description and Figure 2, as a weighting factor, the model probability $\mu^{(i)}[k]$ is important to the interacting/mixing and combination steps. In IMM algorithm, the model probabilities are given by

$$
\mu^{(i)}[k]=\frac{1}{c[k]} \sum_{j} \Gamma^{(j i)} \cdot \mu^{(j)}[k-1] \cdot \mathcal{N}\left[\mathbf{u}^{(j)}[k] ; 0, \mathbf{S}_{K}^{(j)}[k]\right],
$$




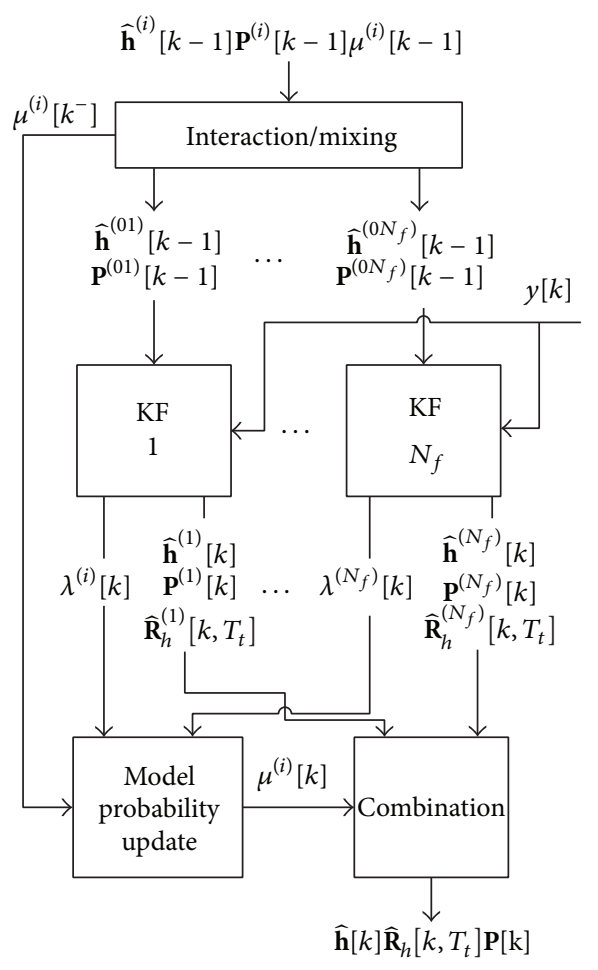

FIGURE 2: Structure of IMM channel estimation.

where $c[k]$ is the normalization constant and $\mathcal{N}\left[\mathbf{u}^{(j)}[k]\right.$; $\left.0, S_{K}^{(j)}[k]\right]$ denotes the probability of $\mathbf{u}^{(j)}[k]$ in the normal distribution with zero mean and the covariance of $S_{K}^{(i)}[k]$. $\mathbf{u}^{(j)}[k]$ and $\mathbf{S}_{K}^{(j)}[k]$ are the residual and its covariance at time $k$ for the model $j$ based KF and illustrated in the summary of IMM based channel estimator in Appendix B.

3.3. Discussions. The data format suitable for IMM based channel estimation algorithm on the basis of single carrier communication system is shown in Figure 3(a). Training sequences are sent frequently. The time interval between the two training sequences is $T_{t}$, with the assumption that the channel correlation $\mathbf{R}_{h}\left[k, T_{t}\right]$ is big enough and the influence of CIR variance during $T_{t}$ can be omitted. In an adaptive communication system, $\mathbf{R}_{h}\left[k, T_{t}\right]$, which reflects the speed of channel variance, can be used to adapt the transmitter. And $T_{t}$ can be adjusted according to the channel correlation to achieve better reliability. Moreover, with the help of software radio, adaptive communication can also change its method of communication, such as modulation and coding, with respect to the channel correlation.

From the previous analysis, although presented on the basis of single carrier communication systems, the IMM based channel estimation algorithm can also be applied in multiple carrier communication systems, such as OFDM systems in two ways, training mode and pilot mode, whose training symbols are arranged as in Figures 3(b) and 3(c). In training mode, training symbols are sent in all subcarriers at the same time; the IMM channel estimation is functional by setting the elements of matrix $\boldsymbol{S}$ in (9) as the sampled signal at

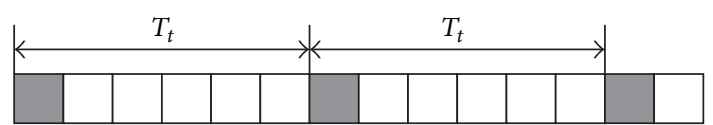

(a) Single carrier communication

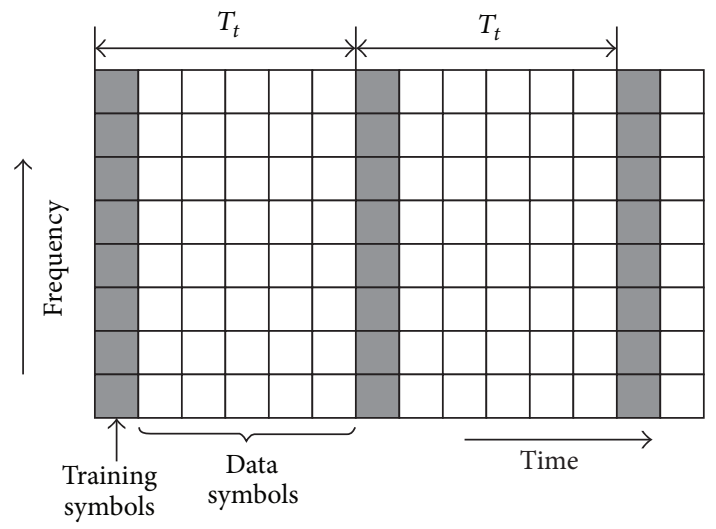

(b) Training mode of multiple carrier communication

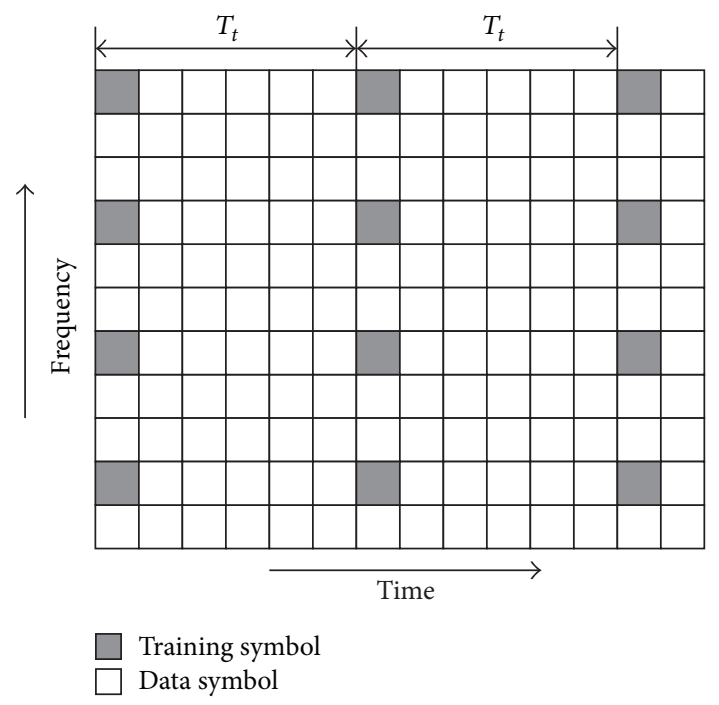

(c) Pilot mode of multiple carrier communication

FIgURE 3: Typical arrangement of training symbols.

the receiver. In pilot mode training symbols are sent in some of the subcarriers with data symbols sent in other subcarriers at the same time. A multiple carrier communication is equivalent to the signal transmitted in parallel. Therefore, IMM based channel estimation can be applied separately in subcarriers with training symbols. In each subcarrier, the wireless channel can be seen as a Rayleigh flat fading channel. The CIR at each subcarrier can be estimated by IMM based channel estimation algorithm, and then these estimates are interpolated via different methods to obtain the general CIR.

\section{Simulation Results and Performance Analysis}

In this section, the performance of both KF and IMM based channel estimation algorithms is compared and analyzed 


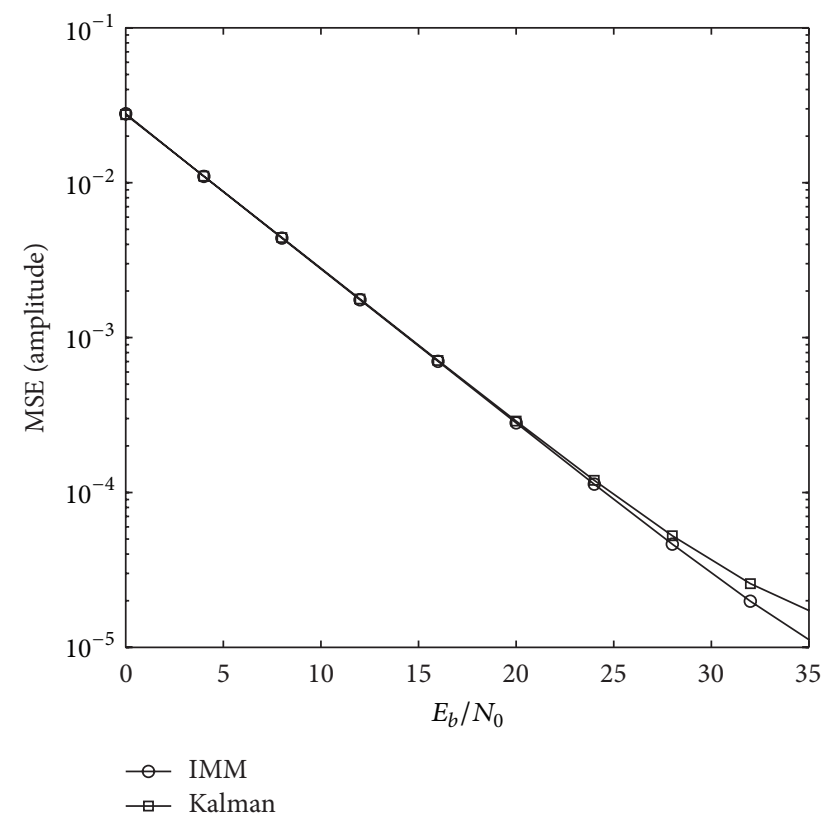

FIGURE 4: MSEs of CIR amplitude with constant maximum Doppler frequency.

based on simulation experiments in different conditions. The channel estimation algorithms are applied to a wireless system whose signal is modulated by 16-QAM with the carrier frequency $2.4 \mathrm{GHz}$ and the symbol period $T_{s}=3 \mu \mathrm{s}$. The signals travel through a Rayleigh frequency flat fading channel, which is estimated every $T_{t}=500 T_{s}$.

In the first case, the maximum Doppler frequency shift of the fading channel is a constant value $f_{\mathrm{d}}=100 \mathrm{~Hz}$. The MSEs of the results from different algorithms are analyzed first. The MSE of an estimated parameter is calculated as

$$
\operatorname{MSE}[\widehat{a}[k]]=\frac{\sum_{K}(a[k]-\widehat{a}[k])^{2}}{K}, \quad k=1, \ldots, K,
$$

where $\widehat{a}[k]$ is the estimated value of $a[k]$ at time $k$. The MSEs of estimated channel amplitude and phase in different measurement noise levels are shown in Figures 4 and 5. Seen from the figures, it can be found that channel estimation based on IMM performs better than the KF estimator in MSE. The reason is that IMM estimator operates in one model with the model probability of 1 , when $f_{\mathrm{d}}$ is constant. The estimated value is more accurate than KF when the model is set precisely. A similar result can be found in BER which is shown in Figure 6. Figure 6 is the relation between BER and $E_{b} / N_{0}$ with different channel estimation algorithms applied in the receiver. It can be concluded that the channel estimation algorithm with better MSE performance can lead to a better BER performance.

In the second case, the maximum Doppler shift $f_{\mathrm{d}}$ switches between $100 \mathrm{~Hz}$ and $200 \mathrm{~Hz}$ every $150 \mathrm{~ms}$. The IMM channel estimator is carried using two models $\left(f_{\mathrm{d}}^{(1)}=100 \mathrm{~Hz}\right.$,

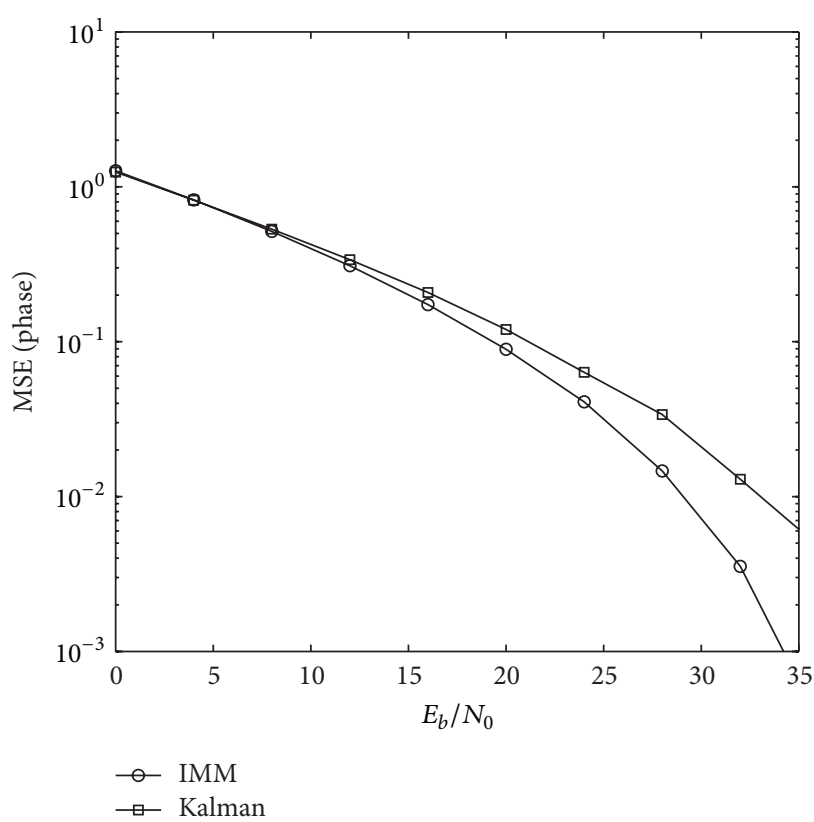

FIGURE 5: MSEs of CIR phase with constant maximum Doppler frequency.

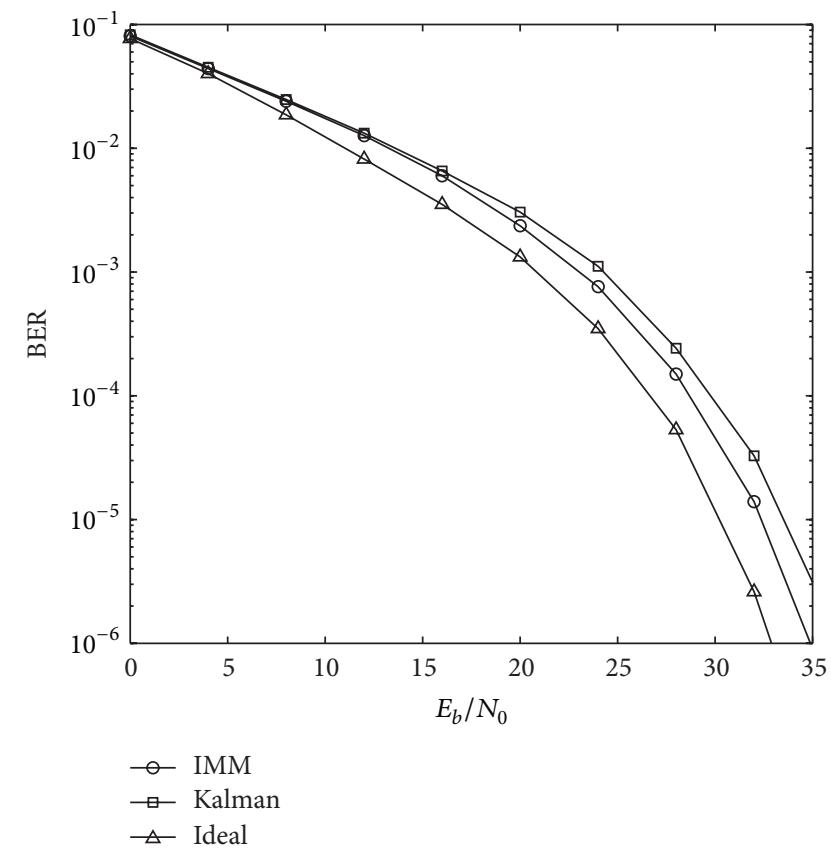

Figure 6: Bit error rates with constant maximum Doppler frequency.

$f_{\mathrm{d}}^{(2)}=200 \mathrm{~Hz}$ ) governed by the Markov chain transition matrix between models:

$$
\Gamma=\left[\begin{array}{cc}
0.993 & 0.007 \\
0.01 & 0.99
\end{array}\right] .
$$

The channel correlation $\mathbf{R}_{h}\left[k, T_{t}\right]$ is analyzed first. Figure 7 shows the change of the real and estimated $\mathbf{R}_{h}\left[k, T_{t}\right]$ 


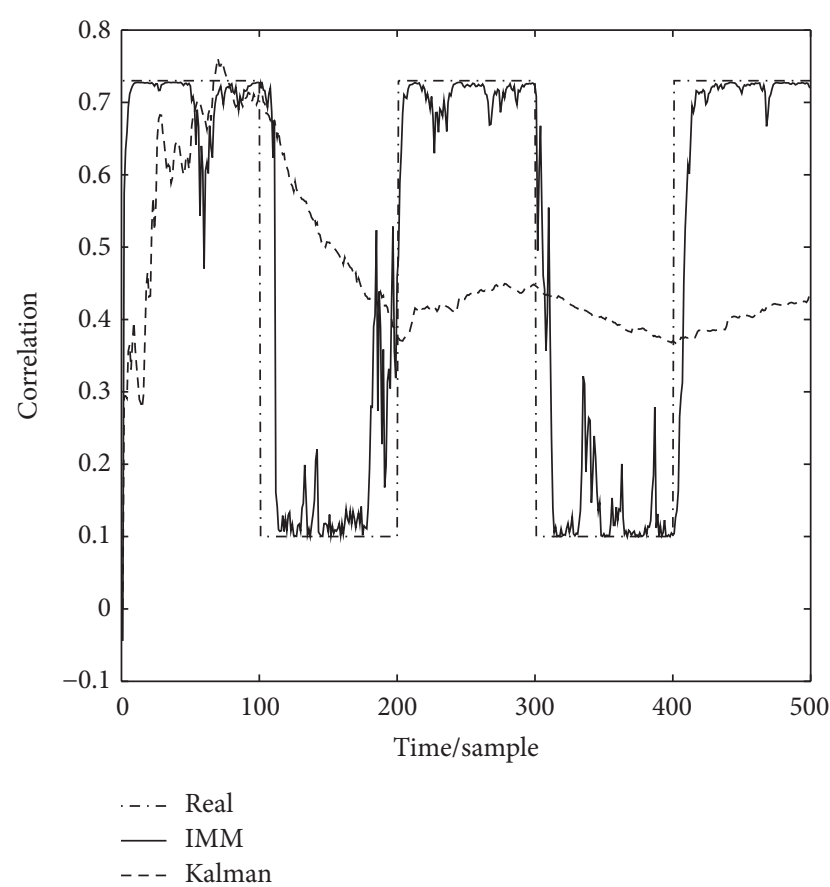

Figure 7: Real and estimated channel correlations.

by different channel estimation algorithms without measurement noise $\mathbf{w}[k]$ presenting. Seen from the figure, KF cannot provide accurate $\mathbf{R}_{h}\left[k, T_{t}\right]$. Equation (17) shows that the estimated processing noise covariance used in $\mathrm{KF}$ is an average of historical observed values. Since channel correlation is related to processing noise covariance, KF cannot track time-variant $\mathbf{R}_{h}\left[k, T_{t}\right]$ precisely. In contrast, based on IMM algorithm, the channel correlation can be estimated successfully as shown in Figure 7. The MSEs of real and estimated $\mathbf{R}_{h}\left[k, T_{t}\right]$ with different measurement noise levels are shown in Figure 8. Seen from Figures 7 and 8, IMM channel estimation algorithm can provide accurate channel correlation $\mathbf{R}_{h}\left[k, T_{t}\right]$, which not only affects the performance of the channel estimation algorithm but is also important information for the adaptive communication system to adjust the system parameters. In contrast the KF based estimator fails to estimate the time-variant channel correlation.

The MSE of CIR is also analyzed in this case. The MSEs of amplitude and phase are shown in Figures 9 and 10. Similarly, as the previous case, with accurate estimated $\mathbf{R}_{h}\left[k, T_{t}\right]$, IMM channel estimation performs better than KF based channel estimators. Contrasting with Figures 4 and 5 in the first case, the KF performs much worse when $\mathbf{R}_{h}\left[k, T_{t}\right]$ is time-variant, because a reliable time-variant $\mathbf{R}_{h}\left[k, T_{t}\right]$ cannot be obtained by KF. Consequently, the BER performances of the algorithms are affected by the accuracy of the estimated CIRs. Figure 11 shows the BERs in different measurement noise levels. It is the same as the pervious analysis that the IMM channel estimation algorithm performs better than the KF method in BER when $\mathbf{R}_{h}\left[k, T_{t}\right]$ changes in time.

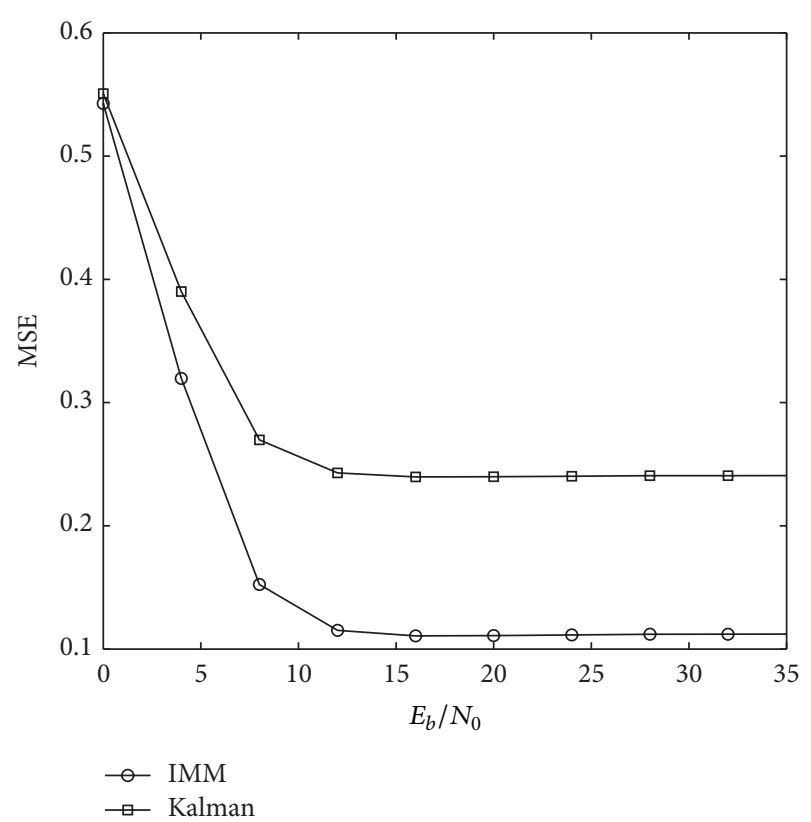

FIGURE 8: MSEs of channel correlation with time-variant maximum Doppler frequency.

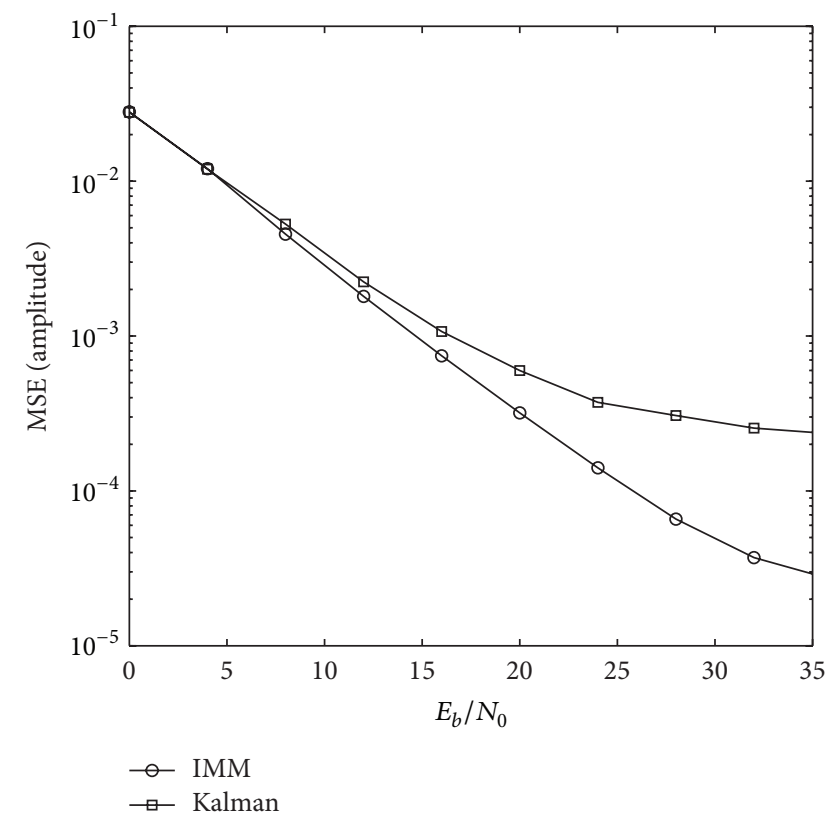

FIGURE 9: MSEs of CIR amplitude with time-variant maximum Doppler frequency.

\section{Summary and Conclusion}

Correlation of a fading channel is important information for channel estimation. The performance of traditional channel estimation with the consideration of time-variance channel correlation is limited. In this paper, by modeling wireless fading channel as a hybrid dynamic system, the channel estimation algorithm based on IMM is presented. The proposed algorithm can identify the channel correlation with the help 


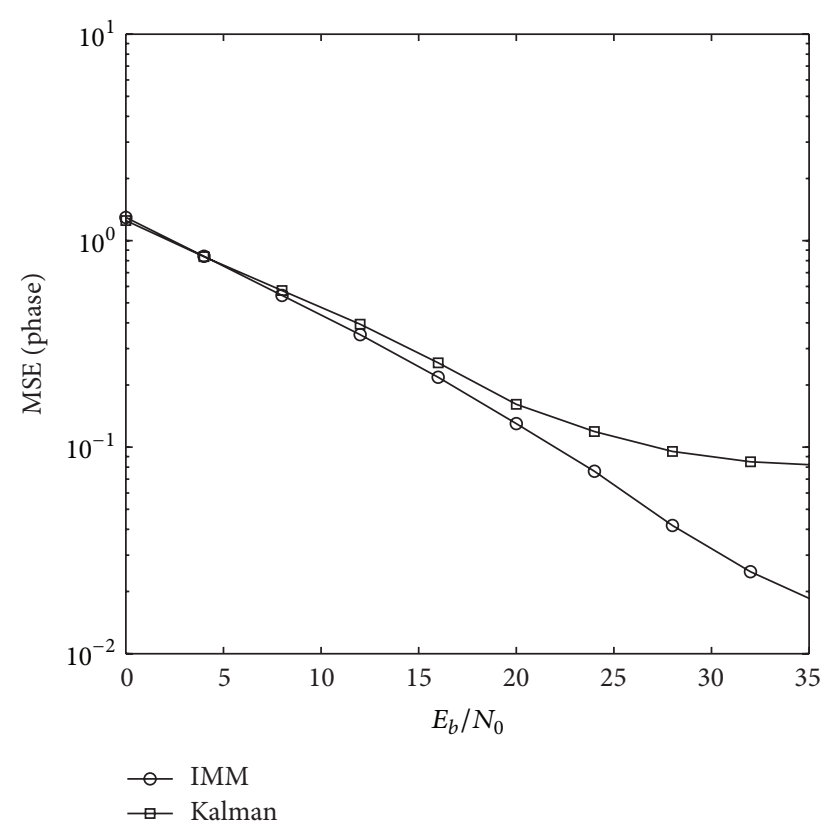

FIGURE 10: MSEs of CIR phase with time-variant maximum Doppler frequency.

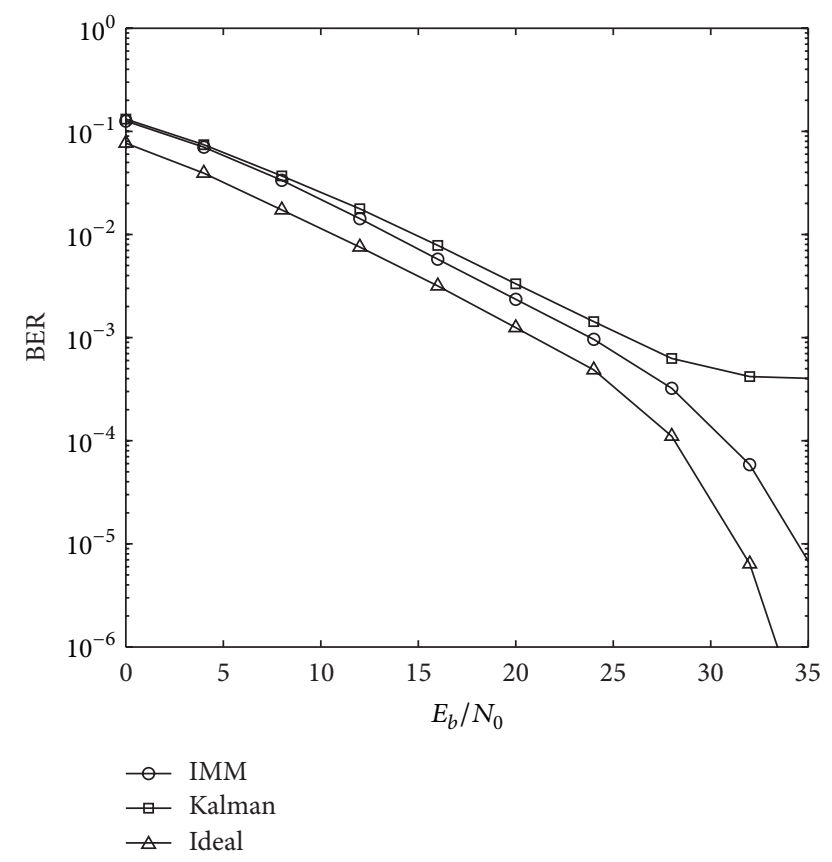

FIGURE 11: Bit error rates with time-variant maximum Doppler frequency.

of the IMM algorithm. With accurate information of channel correlation, the proposed algorithm is capable of performing accurate estimation on the fading wireless channel with timevariant or time-invariant correlation. The results of a number of simulation experiments show that the proposed algorithm is efficient with good performance in estimating CIR as well as the channel correlation.

\section{Appendices}

\section{A. Derivation of the Process Noise Covariance}

From (4), the following equation can be obtained:

$$
\begin{aligned}
\mathbf{h}[k] \cdot \mathbf{h}^{*}[k]= & \mathbf{h}[k-1] \cdot \mathbf{h}^{*}[k-1]+\mathbf{h}[k-1] \\
& \cdot \mathbf{v}^{*}[k]+\mathbf{v}[k] \cdot \mathbf{h}^{*}[k-1]+\mathbf{v}[k] \cdot \mathbf{v}^{*}[k] .
\end{aligned}
$$

Calculating the expectation and taking (10) and (20) into the upper equation,

$$
\begin{aligned}
\mathbf{E}_{h}[k]= & \mathrm{E}\left[\mathbf{h}[k] \cdot \mathbf{h}^{*}[k]\right] \\
= & \mathrm{E}\left[\mathbf{h}[k-1] \cdot \mathbf{h}^{*}[k-1]\right]+\mathrm{E}\left[\mathbf{h}[k-1] \cdot \mathbf{v}^{*}[k]\right] \\
& +\mathrm{E}\left[\mathbf{v}[k] \cdot \mathbf{h}^{*}[k-1]\right]+\mathrm{E}\left[\mathbf{v}[k] \cdot \mathbf{v}^{*}[k]\right] \\
= & \mathbf{E}_{h}[k]+\mathrm{E}\left[\mathbf{h}[k-1] \cdot \mathbf{v}^{*}[k]\right] \\
& +\mathrm{E}\left[\mathbf{v}[k] \cdot \mathbf{h}^{*}[k-1]\right]+\mathbf{Q}[k] .
\end{aligned}
$$

The following equation can be obtained from (A.2):

$$
\mathrm{E}\left[\mathbf{h}[k-1] \cdot \mathbf{v}^{*}[k]\right]+\mathrm{E}\left[\mathbf{v}[k] \cdot \mathbf{h}^{*}[k-1]\right]+\mathbf{Q}[k]=0 .
$$

Taking (19) into (A.3), $\mathbf{R}_{h}\left[k, T_{t}\right]$ can be written as

$$
\begin{aligned}
\mathbf{R}_{h}\left[k, T_{t}\right] & =\mathrm{E}\left[\mathbf{h}[k] \cdot \mathbf{h}^{*}[k-1]\right] \\
& =\mathrm{E}\left[(\mathbf{h}[k-1]+\mathbf{v}[k]) \cdot \mathbf{h}^{*}[k-1]\right] \\
& =\mathbf{E}_{h}[k]+\mathrm{E}\left[\mathbf{v}[k] \cdot \mathbf{h}^{*}[k-1]\right] .
\end{aligned}
$$

Taking (A.3) into (A.4), the covariance of the process noise $\mathbf{v}[k]$ can be written in the form

$\mathbf{Q}[k]=\left(\mathbf{E}_{h}[k]+\mathbf{E}_{h}^{*}[k]\right)-\left(\mathbf{R}_{h}\left[k, T_{t}\right]+\mathbf{R}_{h}^{*}\left[k, T_{t}\right]\right)$.

\section{B. Summary of the IMM Based Channel Estimator}

(1) Interaction/Mixing. For each model $i$, we get the following. Predicted model probability:

$\mu^{(i)}\left[k^{-}\right] \triangleq \operatorname{Pr}\left\{f_{\mathrm{d}}^{(i)}[k] \mid \mathbf{Y}^{k}\right\}=\sum_{j} \Gamma^{(j i)} \mu^{(j)}[k-1]$.

Mixing probability:

$$
\begin{aligned}
\mu^{(j \mid i)}\left[k^{-}\right] & \triangleq \operatorname{Pr}\left\{f_{\mathrm{d}}^{(j)}[k-1] \mid f_{\mathrm{d}}^{(i)}[k], \mathbf{Y}^{k-1}\right\} \\
& =\frac{\Gamma^{(j i)} \mu^{(j)}[k-1]}{\mu^{(i)}\left[k^{-}\right]} .
\end{aligned}
$$

Mixing estimate:

$$
\widehat{\mathbf{h}}^{(0 i)}[k-1] \triangleq \sum_{j} \widehat{\mathbf{h}}^{(j)}[k-1] \mu^{(j \mid i)}\left[k^{-}\right] .
$$


Mixing covariance:

$$
\mathbf{P}^{(0 i)}[k-1]=\sum_{j} \mathbf{P}^{(j)}[k-1] \mu^{(j \mid i)}\left[k^{-}\right]+\mathbf{X}^{(i)},
$$

where

$$
\begin{aligned}
\mathbf{X}^{(i)} \triangleq \sum_{j} & \left(\mathbf{h}^{(j)}[k-1]-\widehat{\mathbf{h}}^{(0 i)}[k-1]\right) \\
& \cdot\left(\mathbf{h}^{(j)}[k-1]-\widehat{\mathbf{h}}^{(0 i)}[k-1]\right)^{\mathrm{T}} \mu^{(j \mid i)}\left[k^{-}\right] .
\end{aligned}
$$

(2) Kalman Filtering. For each model $i$, we get the following. 1] as

Separating the real and image parts, we can write $\widehat{\mathbf{h}}^{(0 i)}[k-$

$$
\widehat{\mathbf{h}}_{\mathrm{ex}}^{(0 i)}[k-1]=\left\{\operatorname{Re}\left\{\widehat{\mathbf{h}}^{(0 i)}[k-1]\right\}, \operatorname{Im}\left\{\widehat{\mathbf{h}}^{(0 i)}[k-1]\right\}\right\}^{\mathrm{T}} .
$$

Predicted mean and covariance:

$$
\begin{aligned}
\widehat{\mathbf{h}}_{\mathrm{ex}}^{(i)}\left[k^{-}\right] & =\widehat{\mathbf{h}}_{\mathrm{ex}}^{(0 i)}[k-1] \\
\mathbf{P}^{(i)}\left[k^{-}\right] & =\mathbf{P}^{(0 i)}[k-1]+\mathbf{Q}_{\mathrm{ex}}^{(i)}[k-1],
\end{aligned}
$$

where

$$
\mathbf{Q}^{(i)}[k]=2 \mathbf{E}_{h}[k]-2 \mathbf{R}_{h}^{(i)}\left[k, T_{t}\right] .
$$

Residual and Kalman gain:

$$
\begin{aligned}
& \mathbf{u}^{(i)}[k] \triangleq \mathbf{y}_{\mathrm{ex}}[k]-\mathbf{D}_{\mathrm{ex}} \widehat{\mathbf{h}}^{(i)}\left[k^{-}\right], \\
& \mathbf{S}_{K}^{(i)}[k]=\mathbf{D}_{\mathrm{ex}} \mathbf{P}^{(i)}\left[k^{-}\right] \mathbf{D}_{\mathrm{ex}}^{\mathrm{T}}+\mathbf{R}[k], \\
& \mathbf{K}^{(i)}[k]=\mathbf{P}^{(i)}\left[k^{-}\right] \mathbf{D}_{\mathrm{ex}}^{\mathrm{T}}\left(\mathbf{S}_{K}^{(i)}[k]\right)^{-1},
\end{aligned}
$$

where $\mathbf{D}_{\mathrm{ex}}$ is the Jacobian matrix of the measurement equation described by (13).

Update state and covariance matrix:

$$
\begin{aligned}
\widehat{\mathbf{h}}_{\mathrm{ex}}^{(i)}[k] & =\widehat{\mathbf{h}}_{\mathrm{ex}}^{(i)}\left[k^{-}\right]+\mathbf{K}^{(i)}[k] \mathbf{u}^{(i)}[k], \\
\widehat{\mathbf{h}}^{(i)}[k] & =\mathbf{M} \widehat{\mathbf{h}}_{\mathrm{ex}}^{(i)}[k], \\
\mathbf{P}^{(i)}[k] & =\mathbf{P}^{(i)}\left[k^{-}\right]-\mathbf{K}^{(i)}[k] \mathbf{D}_{\mathrm{ex}} \mathbf{P}^{(i)}\left[k^{-}\right],
\end{aligned}
$$

where $\mathbf{M}$ is defined as

$$
\mathbf{M} \triangleq\left[\mathbf{I}_{L}, i \mathbf{I}_{L}\right]
$$

where $\mathbf{I}_{L}$ is $L \times L$ identity matrix. Likelihood function:

$$
\lambda^{(i)}[k]=\mathcal{N}\left[\mathbf{u}^{(i)}[k] ; 0, \mathbf{S}_{K}^{(i)}[k]\right] .
$$

Model probability:

$$
\mu^{(i)}[k]=\frac{\mu^{(i)}\left[k^{-}\right] \lambda^{(i)}[k]}{\sum_{j} \mu^{(j)}\left[k^{-}\right] \lambda^{(j)}[k]} .
$$

(3) Combination. Estimated state and covariance matrix:

$$
\begin{aligned}
& \widehat{\mathbf{h}}[k]=\sum_{i} \widehat{\mathbf{h}}^{(i)}[k] \cdot \mu^{(i)}[k], \\
& \mathbf{P}[k]=\sum_{i} \mathbf{P}^{(i)}[k] \mu^{(i)}[k]+\mathbf{X},
\end{aligned}
$$

where

$$
\mathbf{X} \triangleq \sum_{i}\left(\widehat{\mathbf{h}}_{\mathrm{ex}}^{(i)}[k]-\widehat{\mathbf{h}}_{\mathrm{ex}}[k]\right)\left(\widehat{\mathbf{h}}_{\mathrm{ex}}^{(i)}[k]-\widehat{\mathbf{h}}_{\mathrm{ex}}[k]\right)^{\mathrm{T}} \mu^{(i)}[k] .
$$

Estimated correlation matrix:

$$
\widehat{\mathbf{R}}_{h}\left[k, T_{t}\right]=\sum_{i} \widehat{\mathbf{R}}_{h}^{(i)}\left[k, T_{t}\right] \mu^{(i)}[k] .
$$

\section{Conflict of Interests}

The authors declare that there is no conflict of interests regarding the publication of this paper.

\section{Acknowledgments}

This work was supported by the National Natural Science Foundation of China (Grant no. 61240007), Natural Science Foundation of Heilongjiang Province of China (Grant no. F201337), and Research Foundation of National Police University of China. The authors would like to thank Professor Richard Yu and Professor Geoffrey G. Messier for helpful discussions and useful suggestions.

\section{References}

[1] S. G. Kang, Y. M. Ha, and E. K. Joo, "A comparative investigation on channel estimation algorithms for OFDM in mobile communications," IEEE Transactions on Broadcasting, vol. 49, no. 2, pp. 142-149, 2003.

[2] O. Edfors, M. Sandell, J.-J. D. Van Beek, S. K. Wilson, and P. O. Börjesson, "OFDM channel estimation by singular value decomposition," IEEE Transactions on Communications, vol. 46, no. 7, pp. 931-939, 1998.

[3] M. Morelli and U. Mengali, "A comparison of pilot-aided channel estimation methods for OFDM systems," IEEE Transactions on Signal Processing, vol. 49, no. 12, pp. 3065-3073, 2001.

[4] S. Challa, M. R. Morelande, D. Mušicki, and R. J. Evans, Fundamentals of Object Tracking, Cambridge University Press, Cambridge, UK, 2011.

[5] K.-Y. Han, S.-W. Lee, J.-S. Lim, and K.-M. Sung, "Channel estimation for OFDM with fast fading channels by modified Kalman filter," IEEE Transactions on Consumer Electronics, vol. 50, no. 2, pp. 443-449, 2004.

[6] C. Komninakis, C. Fragouli, A. H. Sayed, and R. D. Wesel, "Multi-input multi-output fading channel tracking and equalization using Kalman estimation," IEEE Transactions on Signal Processing, vol. 50, no. 5, pp. 1065-1076, 2002.

[7] A. Visakh and N. Upadhyay, "Channel estimation for OFDM systems using Kalman filter algorithm," in Proceedings of the 1st International Conference on Wireless Technologies for Humanitarian Relief (ACWR '11), pp. 49-52, ACM, Kerala, India, December 2011. 
[8] Y. Li, "Pilot-symbol-aided channel estimation for OFDM in wireless systems," IEEE Transactions on Vehicular Technology, vol. 49, no. 4, pp. 1207-1215, 2000.

[9] Z. Tang, R. C. Cannizzaro, G. Leus, and P. Banelli, "Pilotassisted time-varying channel estimation for OFDM systems," IEEE Transactions on Signal Processing, vol. 55, no. 5, pp. 22262238, 2007.

[10] E. Mazor, A. Averbuch, Y. Bar-Shalom, and J. Dayan, "Interacting multiple model methods in target tracking: a survey," IEEE Transactions on Aerospace and Electronic Systems, vol. 34, no. 1, pp. 103-123, 1998.

[11] X. R. Li and Y. Bar-Shalom, "A recursive multiple model approach to noise identification," IEEE Transactions on Aerospace and Electronic Systems, vol. 30, no. 3, pp. 671-684, 1994.

[12] H. Wu and H. Ye, "State estimation for networked systems: an extended IMM algorithm," International Journal of Systems Science, vol. 44, no. 7, pp. 1274-1289, 2013.

[13] C. Ramesh and V. Vaidehi, "IMM-based Kalman filter for channel estimation in UWB OFDM systems," International Journal of Electronics and Communications, vol. 61, no. 8, pp. 509-514, 2007.

[14] P. Bello, "Characterization of randomly time-variant linear channels," IEEE Transactions on Communications, vol. 11, no. 4, pp. 360-393, 1963.

[15] Y.-S. Choi, P. J. Voltz, and F. A. Cassara, "On channel estimation and detection for multicarrier signals in fast and selective Rayleigh fading channels," IEEE Transactions on Communications, vol. 49, no. 8, pp. 1375-1387, 2001.

[16] X. R. Li and V. P. Jilkov, "Survey of maneuvering target tracking. Part V. Multiple-model methods," IEEE Transactions on Aerospace and Electronic Systems, vol. 41, no. 4, pp. 1255-1321, 2005.

[17] Y. Bar-Shalom and T. E. Fortmann, Tracking and Data Association, Academic Press, 1988. 


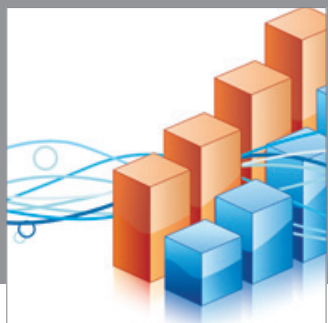

Advances in

Operations Research

mansans

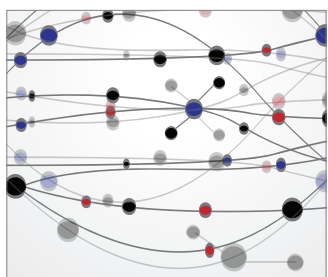

The Scientific World Journal
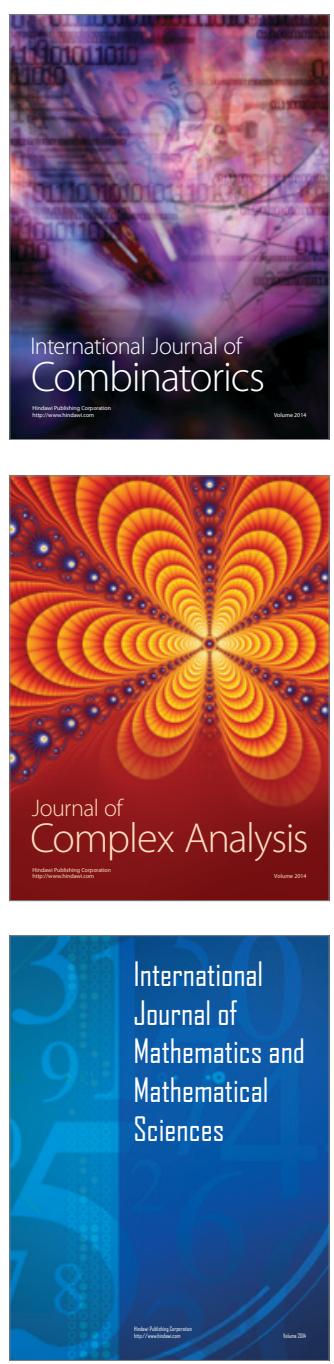
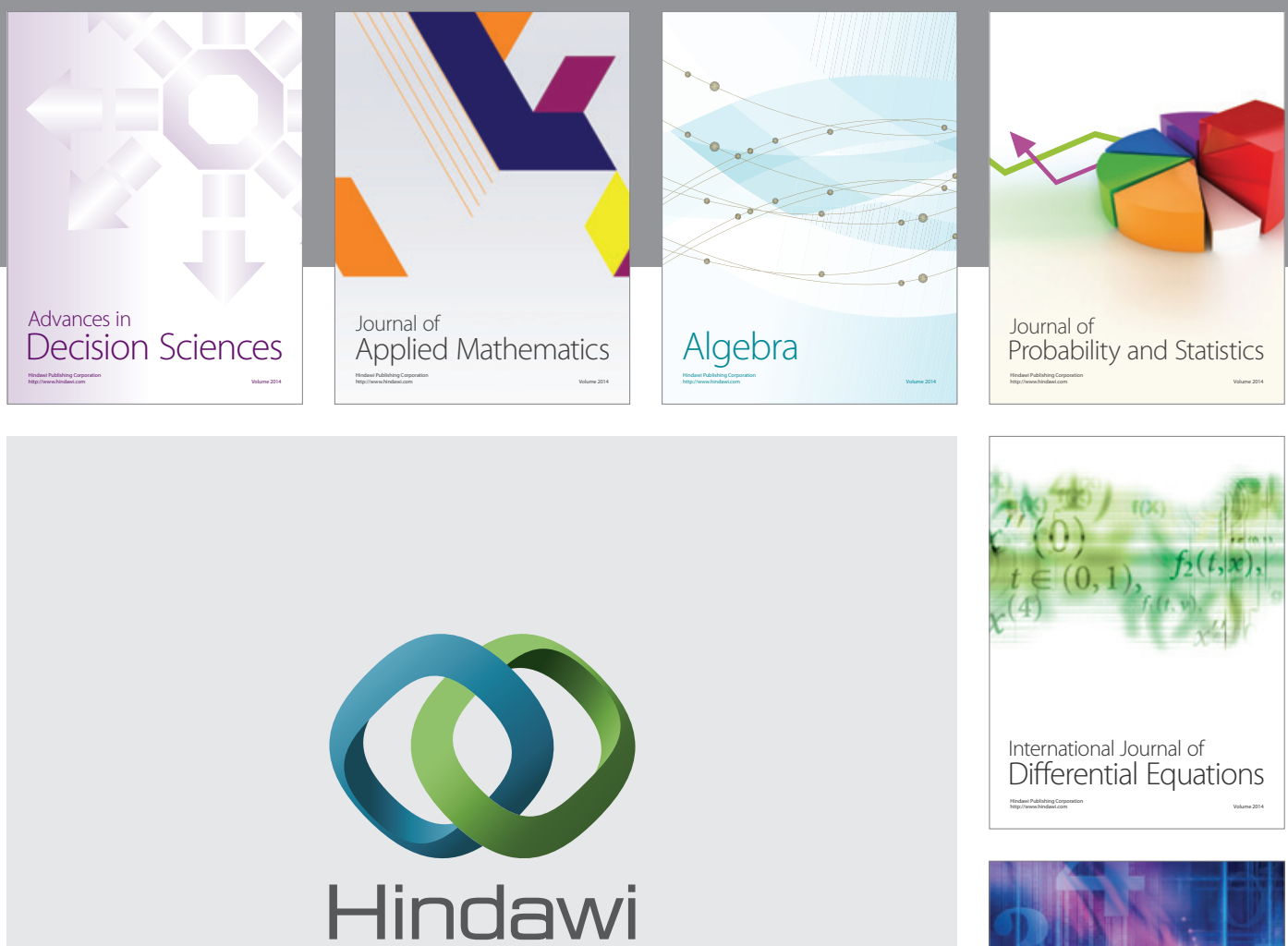

Submit your manuscripts at http://www.hindawi.com
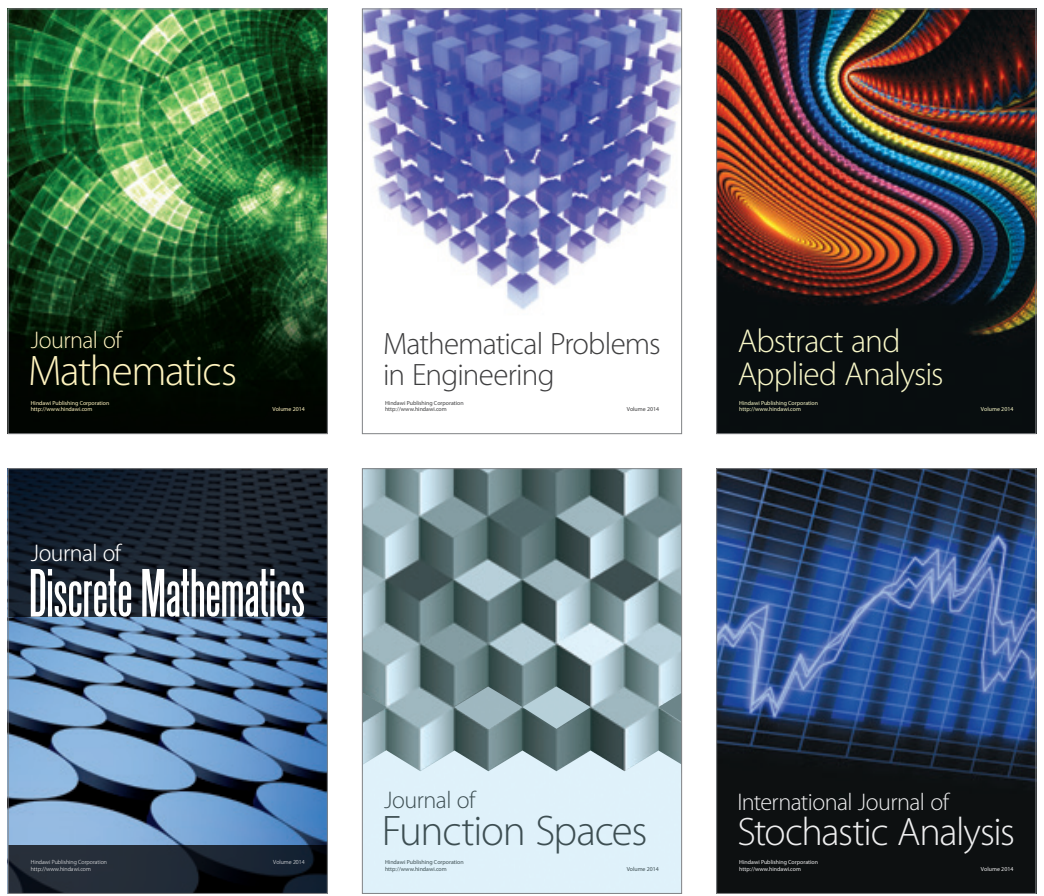

Journal of

Function Spaces

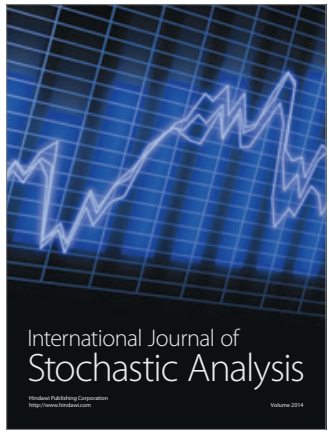

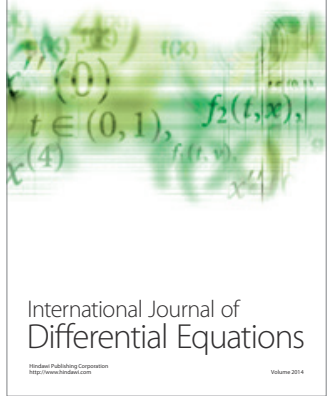
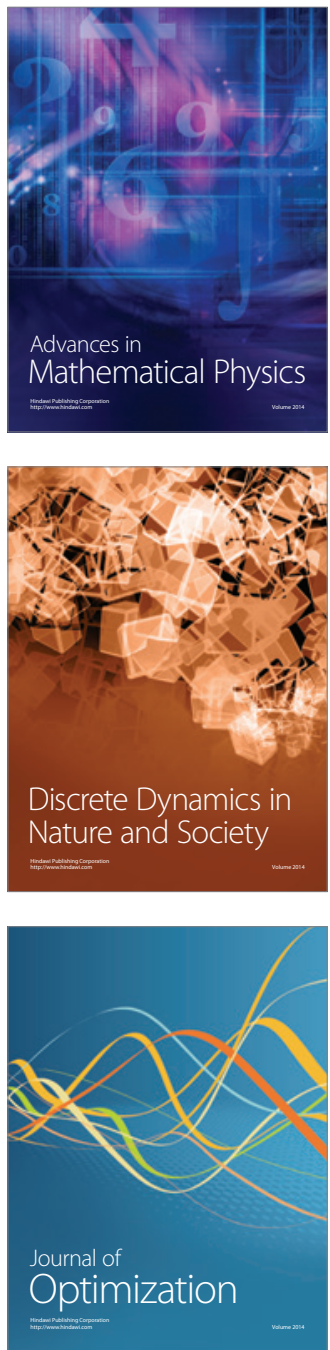